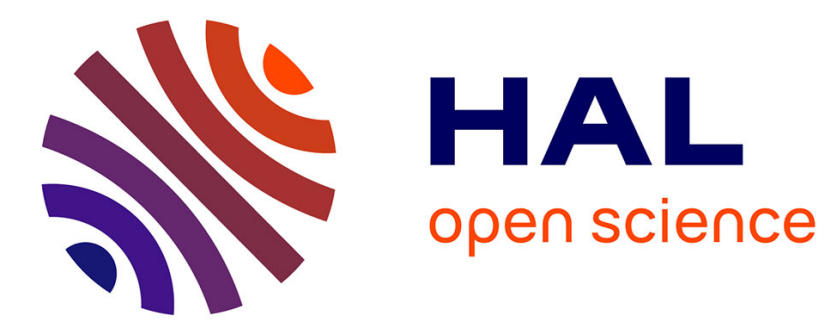

\title{
Le chapitre "vie et mort" du Thésurus de l'œil de la vraie loi de Dōgen

\author{
Frédéric Girard
}

\section{To cite this version:}

Frédéric Girard. Le chapitre "vie et mort" du Thésurus de l'œil de la vraie loi de Dōgen. Cahiers d'Extrême-Asie, 1996, no. 9, p. 299-311. halshs-03134252

\section{HAL Id: halshs-03134252 \\ https://shs.hal.science/halshs-03134252}

Submitted on 8 Feb 2021

HAL is a multi-disciplinary open access archive for the deposit and dissemination of scientific research documents, whether they are published or not. The documents may come from teaching and research institutions in France or abroad, or from public or private research centers.
L'archive ouverte pluridisciplinaire HAL, est destinée au dépôt et à la diffusion de documents scientifiques de niveau recherche, publiés ou non, émanant des établissements d'enseignement et de recherche français ou étrangers, des laboratoires publics ou privés. 


\title{
Le chapitre "vie et mort" du Thésurus de l'œil de la vraie loi de
}

\section{Dōgen}

\section{Frédéric Girard}

\begin{abstract}
The "Shōbōgenzō" preserved two sermons about life and death. The first, "Total operation" (1242), was written by Dōgen when he was fully mature. It is a treatise of a very intellectual type, discussing an active immanent process which holds the world to be a manifestation of the Buddha.

The second one, "Life and death," of unknown date, was integrated much later, in the Ashikaga period, in a work called "The Secret Thesaurus" in the Edo period. The doctrine expounded shows affinity to those of "Total operation": the cycle of life and death is seen as constitutive of the life of the Buddha, as in other sermons in the "Thesaurus." Nevertheless, the idea of an "extremely easy way," a quietist faith, and the fluid, non-rhetorical style, surprise us with their tone, so different from most of the other sermons. It has been thought that this sermon might be a fake, or a text written for a layperson, possibly even for the great Amidist Shinran. It also has been seen as a profession of faith from Dōgen himself. While other of his sermons reveal an essentially intellectual agnosticism, this one reflects a devotional pietism. Does this indicate the possibility that there were two separate individuals named of Dōgen, or rather was there an evolution in the thought the founder of Sōtô?

At this time it seems impossible to choose between these two possibilities. In addition, it seems certain that this sermon is not part of the "Thesaurus. " Its interest lies in the fact that it brings to the exegete questions about interpretation of Dōgen 's work as well as questions about history of the compilation of his "Grand OEuvre"?
\end{abstract}

\section{Citer ce document / Cite this document :}

Girard Frédéric. Le chapitre "vie et mort" du Thésurus de l'œil de la vraie loi de Dōgen. In: Cahiers d'Extrême-Asie, vol. 9, 1996. Mémorial Anna Seidel. Religions traditionnelles d'Asie orientale. Tome II. pp. 299-311;

doi : https://doi.org/10.3406/asie.1996.1121

https://www.persee.fr/doc/asie_0766-1177_1996_num_9_1_1121

Fichier pdf généré le 06/02/2019 


\title{
LE CHAPITRE “VIE ET MORT” DU THÉSAURUS DE L'CEIL DE LA VRAIE LOI DE DŌGEN
}

\author{
Frédéric GIRARD
}

\begin{abstract}
The "Shöbogenzo" preserved two sermons about life and death. The first, "Total operation" (1242), was written by Dogen when he was fully mature. It is a treatise of very intellectual type, discussing an active immanent process which holds the world to be a manifestation of the Buddha.

The second one, "Life and death," of unknown date, was integrated much later, in the Ashikaga period, in a work called "The Secret Thesaurus" in the Edo period. The doctrine expounded shows affinity to those of "Total operation": the cycle of life and death is seen as constitutive of the life of the Buddha, as in other sermons in the "Thesaurus." Nevertheless, the idea of an "extremely easy way," a quietist faith, and the fluid, non-rhetorical style, surprise us with their tone, so different from most of the other sermons. It has been thought that this sermon might be a fake, or a text written for a layperson, possibly even for the great Amidist Shinran. It also has been seen as a profession of faith from Dōgen himself. While others of his sermons reveal an essentially intellectual agnosticism, this one reflects a devotional pietism. Does this indicate the possibility that there were two separate individuals named of Dogen, or rather was there an evolution in the thought the founder of Söto.?

At this time it seems impossible to choose between these two possibilities. In addition, it seems certain that this sermon is not part of the "Thesaurus." Its interest lies in the fact that it brings to the exegete questions about interpretation of Dögen's work as well as questions about history of the compilation of his "Grand Euvre"?
\end{abstract}

Dōgen 道元 (1200-1253) est l'un des penseurs bouddhiques qui ont réussi à développer un ensemble doctrinal finement élaboré. Il a hérité d'une problématique traditionnelle, formé comme il l'a été dans les temples de Kyōto, dont l'Enryakuji 延暦寺 où l'on enseignait les doctrines dites de l'“éveil foncier”. Mais, après s'être rendu en Chine, il les a repensées dans l'optique du courant Sōtō du Zen 曹洞禪.

Un de ses sermons, “Opération totale" (Zenki 全機, de 1242), se présente comme un court traité sur la vie et la mort. Il y aborde, en fait, le thème de la vie, celui de la mort n'y étant guère traité que comme en contrepoint. Loin d'être une omission, ce silence sur la mort n'indique-t-il pas qu'elle reste une énigme dont on ne peut rien dire, marquant une dimension mystérieuse qui échappe à l'intelligence humaine?

Cahiers d'Extrême-Asie 9 (1996-1997) : 299-311. 
Le rapport qui s'établit entre l'absolu et les choses phénoménales est celui de manifestation à manifesté. La vie et la mort sont comme des expressions de cet absolu, tout en prenant place dans le domaine du relatif et de l'illusoire. La naissance ne marque pas plus un commencement effectif que la mort une fin réelle. $\mathrm{Au}$ fond du procès de la vie et de la mort, œuvre un mécanisme vivant qui se caractérise par son unité passant l'esprit humain. Il actualise tout phénomène, en même temps qu'il œuvre comme une maïeutique libérant de ce cycle. Ce double mouvement de "présentification" et de libération, ce va-et-vient entre le "moteur" du monde et le plan phénoménal, est un des ressorts doctrinaux de ce sermon. Vie et mort sont bien plutôt deux moments et deux phases d'un même acte vital simple comme celui d'étendre et de rétracter le bras. Elles sont la réalité entière prise sous deux aspects différents, et au-delà de laquelle il n'est rien d'autre à chercher. Le monde soumis à la temporalité est le théâtre d'une pulsion motrice émanant d'une grandiose activité, grâce à laquelle il se déploie et se résorbe tour à tour. Cette activité n'est autre que l'unique pensée qui est présente au monde, et qui se confond avec celle du Buddha et de l'éveil' : "La pensée reste constamment; ni le passé ni le présent ne s'y écoulent [ $=$ n'y ont prise]. Prendre conscience de cela c'est se détacher du cycle de la vie et de la mort.",2

La réalité vraie est inscrite dans le monde phénoménal lui-même. Si la philosophie de Dōgen se présente comme une ascèse religieuse et intellectuelle, elle se plaît également à affirmer la vie dans la luxuriance de toutes ses formes, malgré ou d'autant qu'elle est sensibilisée par leur aspect transitoire. C'est la présence immédiate de l'esprit à lui-même et aux choses qui est décrite avec force dans ce sermon. Dōgen tâche d'y mettre en évidence l'intelligibilité inscrite dans le monde. Celui-ci est une vaste aporie à décrypter et comporte une part d'opacité, que l'homme s'efforce de rendre toujours plus transparente en dépassant les vues particlles qu'il en a.

\section{Traduction}

Thésaurus de l'ceil de la vraie Loi 正法眼藏

Volume XXII : “Opération totale 全機”."

"La grande voie des Buddha est à son terme ultime évasion et réalisation."

\footnotetext{
1 "Thésaurus", "Le triple monde est seulement pensée"(1243).

2 "Thésaurus", "Discours sur l'application à la voie"(1231).

"Je suis l'édition de Ōkubo Dōshū 大久保道舟、Dōgen zenji zenshū 道元禪師全集 [CEuvres complètes du maître de dhyāna Dōgen], I, Kyōto, réèd. 1989, pp. 203-205), et fais état de l'essentiel des commentaires dans les notes. Dans zenki 全機, zen 全, “total", veut dire au complet, sans défection; $k i$ 譏 correspond ici à activité, mise en œuvre (hataraki). L'opération totale est une activité stable, qui n'est pas mue par l'être et le non-être, le sensible et le vide, qui est au-delà des six facultés sensorielles ainsi que de leurs objets. Si elle se mouvait d'un côté, il lui manquerait l'autre, et ne pourrait dès lors être qualifiée de totale.

${ }^{+}$Affirmation d'un double mouvement ascensionnel et descendant-selon le Shōbōgenzō
} 
Par évasion, on entend que la vie dépasse la vie et que la mort dépasse la mort. C'est la raison pour laquelle il est une issue hors du cycle de la vie et de la mort ainsi qu'une entrée dans ce même cycle, qui sont en tout point la grande voie ultime. ${ }^{5}$ Il est un détachement de ce cycle ainsi qu'un salut en lui, qui sont tous deux la grande voie ultime. ${ }^{6}$ La réalisation actuelle est vie et la vie est réalisation. Lorsqu'elle est à l'œuvre, la vie n'est pas sans se réaliser entièrement; et il en va de même pour la mort. ${ }^{7}$

Cette opération ${ }^{8}$ permet que la vie et la mort soient. A l'instant précis où cette opération se réalise, elle n'est par nécessité ni grande ni petite, ni ne s'étend au monde entier ni ne reste limitée, n'est ni longue ni courte. ${ }^{9}$

La vie actuelle se trouve dans cette opération et inversement. La vie n'est ni à venir, ni passée, ni actuelle, ni réalisation. Néanmoins, elle réalise bien

monge 正法眼蔵門解 [Explications sur le Thésaurus de l'œil de la vraie loi], version en 95 volumes (abréviation : Monge) de Menzan Zuibō 面山瑞方 (1683-1769) : “Il est deux niveaux pour scruter la grande voie. Tout d'abord, dans la méthode sapientielle, on s'enquiert à fond de toutes choses afin de les dépasser, et dans la méthode de compassion on passe à la réalisation dans le monde entier sans rien faire de caché." (Monge).

"L'entrée dans le cycle transmigratoire est une réintégration de la personne authentique au sein même du relatif : "C'est pourquoi il arrive qu'on sorte du cycle transmigratoire tout comme il se fait qu'on entre dans l'être de la personne réelle, qui est soumise aux allées et venues dans le cycle transmigratoire. Ces deux méthodes scrutent également jusqu'à son terme la grande voie." (Monge).

" "Il est un néant des gens des deux véhicules qui rejettent le cycle transmigratoire, et il est un vœu compatissant des bodhisattva qui sauvent du même cycle. Ces vœux diffèrent certes en profondeur, mais les deux méthodes se rejoignent pour aller jusqu'au bout de la grande voie des Buddha." (Monge).

7 La vie manifeste l'enseignement du Buddha, au travers de Mahākāśyapa qui en est le dépositaire, alors que la mort en est comme l'occultation momentanée et intentionnelle. Selon la parole profonde et intime du Buddha; il y a entre vie et mort la même différence qu'entre actualisation et latence ou explicite et implicite : Quand la vie se manifeste, elle déploie entièrement son activité, et il n'est aucun phénomène ou chose qui ne soit vie-ce à quoi correspond le fait que l'enseignement du Buddha n'est pas resté occulté par Mahākāśyapa-. De même en est-il pour la mort-ce à quoi correspond le fait que le Vénéré du monde a une parole intime de sens profond (mitsugo 密語)_." Monge se réfère ici au sermon "Parole intime" de 1243, qui s'appuie lui-même sur les "Trois cents cas".

${ }^{x}$ Kikan 機閔; les versions Kenkon.in 乾坤院 et Shōbōji 正法寺 lisent ayatsuri, artefax, mise en œuvre.

9 "Etant à la fois transcendante et immanente aux choses, l'opération illustre toutes leurs qualités sans être aucune d'elle non plus. N'étant en tant que telle ni vie ni mort, c'est elle qui fait qu'elles apparaissent ou disparaissent : "Il est une activité qui fait que la vie soit et une autre qui fait que la mort soit, sans qu'on puisse dire qui va vers la vie et qui meurt. La vie et la mort ne sont pas au sein de cette activité. Celle-ci n'est, au départ, ni être ni nonêtre, car elle est vérité de voie médiane. Elle se trouve donc aussi bien dans l'être de la vie que dans le non-être de la mort. Etant donné qu'elle est à part de tous les signes différentiels, elle est à même toutes choses; elle n'est pas sans être le grand et le petit, le long et le court." (Monge). 
l'opération en son entier, tout comme le fait la mort. Avisons-nous que la vie et la mort font partie des innombrables entités qui sont en notre moi. ${ }^{10}$

Il importe de considérer attentivement ce qui suit. Cette vie actuelle ainsi que toutes les entités qui naissent en même temps qu'elle, sont-elles simultanées ou non? On ne voit pas qu'un seul instant du temps ou qu'une seule entité n'accompagne en même temps la vie. On ne voit pas non plus qu'un seul fait ou qu'une seule pensée n'accompagne simultanément la vie.

Prenons un exemple." Imaginons la vie à l'instar d'un homme qui [, comme moi,] serait monté sur un navire. C'est moi qui manœuvre les voiles du vaisseau, qui en prends le gouvernail et rame; et pourtant c'est le navire qui me porte. Mon moi ne lui est pas extérieur. C'est en montant sur lui que je fais en sorte que le bateau soit un bateau. Il importe de bien scruter et examiner ce fait au moment où il survient.

A cet instant précis, le navire [= le moi] n'est jamais sans être le monde :

1" Vie est synonyme d'être, de positivité, de manifestation, et mort de non-être, de négativité et de latence : "Quand cette activité se manifeste du côté de l'être on l'appelle vie; et en déclarant que cette activité s'est manifestée, on n'entend pas par là qu'elle soit nouvellement arrivée." "Mon moi est pourvu des innombrables et infinies entités. Il est porteur, parmi eux, aussi bien des entités de l'être qu'est la vie que de ceux du non-être qu'est la mort." (Monge). L'idée exprimée est la même que celle exposée au début du chapitre “Aporie actualisée" $(1333,1353)$ du "Thésaurus" : le "moi" dćsignc ici non pas l'ego tourné vers lui-même, mais le vrai moi, celui qui est éclairé par la Loi.

"Le navire est une métaphore pour le moi et la vie qui fait être ce moi. Ce moi est libre de disposer de sa vie comme il l'entend, tel le navigateur qui manœuvre la voile. Dans l'image de l'homme monté sur un vaisseau, on compare le bateau à la vie. Il n'est pas de moi en dehors de ce vaisseau dont il dispose à sa guise. C'est en raison du fait que je manœuvre que le moi est vie et que la vie est mon moi; c'est parce que je le manœuvre que le navire est libre. C'est moi-même qui fais que la vie est libre, et tous manœuvrent par l'opération qu'est la vie. Lorsque je vais en haute mer, l'eau et le rivage font partie du monde du vaisseau que je mancuvre. Par vie, on entend que toutes choses sont vie, que la vie et le moi ne sont pas deux et que la vie des êtres et des choses me fait être moi-même. Le corps et la pensée, l'environnement et la personne qu'on a reçus en partage en raison de la rétribution des actes, participent également d'une seule et même vie, grâce au mécanisme opératoire qu'est le vaisseau. Le moi qui est vie et la vie qui définit mon moi ne sont donc pas deux choses différentes. "La personne et le vaisseau sont une même entité et une même vie. La vie et le moi sont intimement liés. Sachons qu'il s'agit d'une comparaison faite avec un objet $(=1 \mathrm{e}$ navire). La traduction chinoise (1765) du “Thésaurus" du commentateur Katsudō Honkō 勝道 本光 (?-1773) porte : “La vie fait que le moi est vie, et le moi fait que la vie est le moi." Il importe de la consulter." (cité dans Shōbōgenzō Shiki 私記 [Notes personnelles sur le Thésaurus...-version en 95 volumes] de Zakke Zōkai 雜華藏海 (1730-1788). En dehors de la vie, il n'est pas de moi. En dehors du moi, il n'est pas de vie. Le moi qu'est le bateau est le navire du moi. En dehors du navire, il n'est pas de moi, et en dehors du moi il n'est pas de navire. Ainsi se laisse définir l'opération qui se réalise totalement. (Shōbōgenzō Naippō 那一宝 [Comm. du Thésaurus... (1791) - version en 95 volumes] de Fuyō Rōran 父 幼老卵 (1723-1805). 
le ciel, le plan d'eau et le rivage appartiennent tous à la temporalité du bateau, je veux dire qu'ils ne sont pas d'un temps distinct de celui du bateau. C'est la raison pour laquelle la vie fait que ma vie soit et que je sois moi. Une fois qu'on est monté sur le navire, le corps et la pensée, l'environnement et l'individualité qu'on a reçus en partage, sont tout uniment l'œuvre du navire; la terre et l'espace entiers sont de même tout uniment l'œuvre du navire. Ainsi sont le moi qui est vie et la vie qui constitue le moi.

Le maître de dhyāna Yuanwu 圆悟, le précepteur Keqin 克勤, ${ }^{12}$ a déclaré : "La vie manifeste entièrement l'opération, et la mort également."

Il convient de tirer au clair et de scruter ce que sous-tend cette assertion. On a à scruter le principe selon lequel la vie manifeste l'opération en son entier : elle est la terre et l'espace en leur entier, sans qu'un début ou une fin lui soient assignés. Or, non seulement cela n'empêche pas non plus que la vie manifeste entièrement l'opération, mais encore n'empêche pas que la mort également la manifeste. Au moment où la mort manifeste entièrement cette opération, elle est la terre ainsi que l'espace en leur entier. Ce qui n'empêche pourtant pas que la mort manifeste entièrement l'opération, sans pour autant que cela fasse obstacle à ce que la vie la manifeste de même entièrement. Aussi bien la vie ne fait-elle pas obstacle à la mort et réciproquement. La terre et l'espace entiers sont ensemble et dans la vie et dans la mort.

Je n'entends toutefois pas par là que la terre entière ou que l'espace entier soient, d'un seul tenant, totalement à l'œuvre soit dans la vie soit dans la mort. Les premiers et les secondes ne sont pas plus uns qu'ils ne sont différents; sans être différents ils ne sont pas non plus identiques; sans être identiques ils n'en sont pas pour autant pluriels. C'est ainsi que dans la vie et dans la mort sont réunis tous les éléments de l'opération totale, et qu'on assiste également à la manifestation de celle-ci dans ce qui n'est ni la vie ni la mort. Il y a et vie et mort dans la manifestation de l'opération totale.

Aussi bien, l'opération entière du cycle de la vie et de la mort est-elle similaire au mouvement d'un jeune homme qui rétracte et allonge le bras, ${ }^{13}$ et

\footnotetext{
${ }^{12}$ Keqin, ou Yuanwu et Foguo 佛果 de ses surnoms (1063-1135), est un disciple de Fayen 法演 (?-1104), le cinquième patriarche de la branche Linji 臨濟, courant Yangqi 楊岐, et le maître de Dahui 大彗 (1089-1163). Il est l'auteur du "Recueil de la falaise smaragdine 碧错 錄” ( $T$. XLVIII, $\mathrm{n}^{\circ}$ 2003), commentaire de cent anecdotes ou cas rassemblés par Xuedou Chongxian 雪䆜重顯 (980-1052). La citation est un commentaire de Keqin à un dialogue sur la vie et la mort entre Daowu 道悟 (769-835) et son disciple Yunyan 雲管 (782-834). Elle illustre l'idée que vie et mort ne sont que des notions relatives, tandis que la véritable nature des choses leur échappe car elle n'apparaît pas plus qu'elle ne disparaît. On la trouve dans le “Recueil de logia du maître de dhyāna Yuanwu Foguo 圆悟佛果禪師語銭” ( T. XLVII, n 1997, p. 793bc). Dōgen a noté ce dialogue dans son "Recueil de trois cents cas", et le commentaire de Keqin dans "Etude de la voie par le corps et la pensée" (1242).

${ }^{13}$ L'image est tirée du "Sūtra de l'examen mental sur Amitāyus", T. XII, n 365, p. 245c.
} 
ressemble-t-elle au geste de quelqu'un qui, en pleine nuit, chercherait son oreiller derrière lui avec la main. ${ }^{14}$

En outre, la réalisation se fait à grand renfort de pouvoirs merveilleux et d'émissions de rayons lumineux [de la part du Buddha]. Au moment précis où elle s'actualise, l'opération est tout entière dans sa réalisation. On sera en conséquence porté à croire qu'il n'y a pas eu de réalisation avant la réalisation en question. On remarquera cependant que, avant ladite réalisation, l'opération tout entière s'était bien manifestée, et qu'elle se soit bien manifestée antérieurement ne fait en rien obstacle à ce que l'opération présente s'actualise entièrement. On voit de la sorte pulluler à qui mieux mieux nombre d'opinions erronées, comme celle dont on vient de faire état.

Thesaurus de l'cil de la vraie Loi, volume XXII : "Opération totale".

Exposé à l'assemblée monacale sous la tente du gouverneur d'Izumo [résidence de Hatano Yoshishige], jouxtant le monastère du Rokuharamitsuji 六波羅蜜寺, province de Yamashiro 山城 [Kyōto], le $17 \mathrm{du} 12^{\mathrm{e}}$ mois de 1242 (Ninji 仁治, 3).

Recopié le 19 du $1^{\text {er }}$ mois de 1243 (Ninji, 4) par Ejō 懁桀."

Dans certaines parties de son œuvre, Dōgen insiste sur le rôle de la pratique, de la foi, de la dévotion ainsi que des “œuvres", qui opèrent au sein d'une moralité religieuse, voire de la grâce du Buddha. Tel semble être le cas d'un sermon de date inconnue, "Vie et mort" (shōji 生死), qui traite du même sujet que le précédent mais en mettant le doigt sur cette part d'inconnaissable.

Le ton du sermon intrigue. Il sonne différemment de la plupart de ceux du "Thésaurus". Aussi est-il l'un des plus controversés : il touche à la cohérence même de la pensée de Dōgen ainsi qu'à l'histoire de la constitution du "Thésaurus". Comme quelques rares autres, tels "De Buddha à Buddha" (copie de 1288) ou "Pensée de la voie" (non daté), il est rédigé dans un style dépouillé et limpide, sans référence érudite ni dialectique verbale ou jeu rhétorique.

${ }^{14}$ Voir le dialogue entre Yunyan et Daowu, noté par Dōgen dans son "Recueil de trois cents cas", $n^{\circ} 105$. Il est extrait du "Recueil de logia du maitre de dhyāna Hongzhi 宏智 禪師黄錄” (T.XLVIII, n² 2001, p. 23b), et du "Recueil de la falaise smaragdine" (Ibidem, $n^{\circ} 2003$, p. $\left.213 \mathrm{c}\right)$.

"Yunyan demanda à Daowu : "Le bodhisattva à la grande compassion (Avalokiteśvara aux mille mains) agit à l'aide de combien de mains et d'yeux?" Daowu répondit : "Il en est comme d'un homme qui chercherait son oreiller derrière lui avec la main." Yuanyan répliqua : "J'ai compris! J'ai compris!" Daowou s'enquit : "Qu'est-ce que tu as compris?" Yuanyan répondit : "C'est son corps entier qui est mains et yeux." Daowou remarqua : "Voilà qui est bien dit. Mais tu as seulement réussi à exprimer les huit ou neuf dixièmes de la vérité." Yuanyan de rétorquer: "Maître, comment comprenez-vous cela?" Daowu de répondre : "De tout son corps il est mains et yeux."» 
Le dialogue attribué à deux moines chinois, qui introduit le sermon, rapporte l'exact contraire de ce qui est dit dans l'original des textes. Cela trahirait-il qu'on a affaire à une version vulgarisée ou retravaillée par un tiers d'un sermon initial? Dōgen a-t-il volontairement procédé à une telle interversion, ce qui ne serait à vrai dire guère pour étonner sous sa plume? En outre, le sermon, qui ne porte pas de colophon, fait partie d'une compilation du "Thésaurus" en 28 volumes dont on ne peut faire remonter la compilation à l'époque de Dōgen : la première copie qu'on en $a$, en trois fascicules, date du début de l'époque Muromachi (fin XIV'-début $X^{e}{ }^{e}$ siècles). Cet ensemble assez composite du "Thésaurus" a été baptisé, selon toute vraisemblance par le $39^{e}$ supérieur du Eiheiji 永平寺, Shōten Sokuchi 承天即地 (?1744), du nom de "Thésaurus secret". On a hésité sur la valeur à attribuer à l'épithète de "secret". S'agit-il d'une transmission "ésotérique" de sermons qu'on ne tenait guère à diffuser en dehors du Eiheiji, ou bien d'une compilation alors tenue "secrète", en raison du fait que le Bakufu avait interdit d'éditer le "Thésaurus" en 1727-il a levé l'interdiction en 1796-1797-? La seconde hypothèse paraît plus plausible. Toujours est-il que c'est de cet ensemble que le $35^{\mathrm{e}}$ supérieur du Eiheiji, Hanjō Kōzen 版橈晃全 (1627-1693), a extrait le chapitre “Vie et mort" pour l'intégrer en 1690 dans la compilation du "Thésaurus" en 95 chapitres. Ce faisceau de données matérielles a fait planer des doutes sur l'attribution de ce texte à Dōgen. On ne peut entièrement les lever, même si l'on admet que la composition de ce sermon n'est pas à dater de l'époque Muromachi, mais de celle du colophon du sermon "De Buddha à Buddha", qui vient clore la version du "Thésaurus secret" et porterait non pas sur ce seul chapitre mais sur l'ensemble de ses 28 chapitres.

A ces éléments, viennent s'ajouter des raisons doctrinales. Dōgen a combattu les tenants de "pratiques faciles"- - les amidistes (?) ${ }^{15}$-, et les adeptes du dhyāna d'une "voic rapide"- de l'école de Bodhidharma (?)—dispensant de toute pratique au nom de la nature pérenne de l'esprit humain ${ }^{16}$ et de l'identité pure et simple entre le moi individuel et le Buddha ${ }^{17}$ - Il a stigmatisé sous le terme d" "hérésie naturaliste" ceux pour qui la connaissance spontanée serait telle quelle l'èveil. ${ }^{18}$ Selon lui, au contraire, la culture est nécessaire afin de mettre en évidence la nature d'éveil qui sommeille en l'homme même si, par ailleurs, la culture de la méditation assise semble ne nécessiter aucune pratique cultuelle impliquant un acte de foi. Or, ne préconise-t-il pas dans ce sermon une "voie extrêment aisée"?

15 "Recueil sur l'application de l'esprit à la culture de la voie" de 1234 (traduction HoangThi-Bich, Etude et traduction du Gakudōyōjin-shū, Recueil de l'application de l'esprit à l'étucle de la voie du maître de zen Dōgen, Droz, Genève-Paris, 1973, pp. 141-144).

16 "Discours sur l'application à la voie" de 1231 (Euvres, I, pp. 738-739; traduction Bernard Faure, Dögen : La vision immédiate, nature, éveil et tradition selon le Shōbōgenzō, Le mail, Paris, 1987, pp. 95-96).

${ }_{17}$ Ihidem, pp. 742-744, trad. Faure, pp. 101-102.

1x "Journal de l'ère Hōkyō", § IV (1225-1227). 
Néanmoins, les partisans de l'authenticité de ce sermon trouvent à l'intégrer dans la pensée de Dōgen. Rien n'y contredit formellement ses positions doctrinales. Il est vrai que, si Dōgen professe la culture de la méditation assise, il y a place également pour des pratiques religieuses ordinaires et le recours à la foi. ${ }^{19} \mathrm{Ne}$ $s$ 'agit-il que de formes inférieures de culte? Si Dōgen l'affirme parfois, ${ }^{20}$ les sermons du "Thésaurus en douze chapitres", qui sont de la dernière période de sa vie, semblent justifier une telle dimension cultuelle de la religion. ${ }^{21}$ Pour lui le lien qui unit le Buddha aux êtres semble revêtir une dimension religieuse : il le compare à celui d'un père compatissant à ses enfants. ${ }^{22}$ C'est en dispensant sa compassion que, grâce à la puissance de ses mérites, il les sauve. En outre, il n'est pas donné à quiconque d'accéder seul à la doctrine et à la pratique vraies; il importe de s'en enquérir auprès d" "amis de bien" afin d'éviter de tomber dans des interprétations entachées de subjectivité et tributaires de la seule lettre des textes. ${ }^{23}$ N'est-ce d'ailleurs pas dans cette optique que l'enseignement a à se transmettre "de Buddha à Buddha"? ${ }^{24}$

On peut aller jusqu'à relever des expressions, comme celle du "timon mis au septentrion pour se diriger au pays de Yue", ou "se laisser œuvrer [par le Buddha]", qui ont des répondants ailleurs. Il est également loisible de considérer que l'expression de "voie extrêmement aisée" ne se réfère en rien aux "pratiques faciles" des amidistes ni aux "voies rapides" du Zen : ne désigne-t-elle pas la méthode de dépouillement qui lui est chère? La méditation assise se définit précisément par un décapage et une voie purgative qui en font l'essentiel. Ce sermon révèlerait un Dōgen moins intellectualiste que quiétiste. Est-ce celui de la

${ }^{19}$ Dōgen n'a pas aboli le culte rendu aux personnages du panthéon, à commencer par Śăkyamuni et Mañjuśri, symbole de la sapience, dont on trouve des représentations dans les monastères Sōtō. Dōgen préconise la salutation au bodhisattva Mañjuśrí ("Méthode d'application à la voie", 1245, et "Méthode pour consommer les repas, les gruaux de riz et le riz", 1246), ainsi que l'invocation de Vairocana, de Saákyamuni, de Maitreya, représentant tous les Buddha, et de Mañjuśrī, Samantabhadra et Avalokiteśvara, représentant tous les bodhisattva ("Méthode pour consommer les repas"). Il semble accorder une place prépondérante à Vairocana, le Buddha du "Sūtra de l'ornementation fleurie". Concernant la pratique selon la foi droite qui permet de réaliser l'éveil quel que soit de degré d'intelligence, v. "Discours", Euvres completes I, p. 745 (traduction Faure, p. 104).

2" V. "Discours", Ibidem.

${ }^{21}$ Ainsi celui intitulé "Culte rendu aux Buddha" (copie de 1255; on considère que ce sermon aurait été prononcé à l'intention de laïcs), préconise-t-il l'offrande comme cause au moins lointaine de la réalisation de l'ćtat de Buddha. De fait, l'offrande authentique est celle de qui se donne corps et âme (Ibidem, p. 656-657).

${ }_{22}^{2}$ "Le triple monde est seulement pensée" (1243); "Discours", Ibidem, p. 741, trad. Faure, p. 99.

2.3 "Discours", Ibidem, p. 730, 731, 735 et 744, traduction Faure, p. 76, 77, 90 et 102: "Recueil", Ibidem, p. 258, traduction Hoang, p. 160.

${ }^{24}$ "Recueil", p. 259, trad. Hoang, p. 167; "Culte rendu aux Buddha". C'est le sujet d'un des sermons de Dōgen (copie de 1288) qui fait partie du "Thésaurus secret", "De Buddha à Buddha". 
maturité qui développe une foi tout humaine? Est-ce le "dernier" Dōgen qui, malade, se rattache à cette foi dénuée de toute spéculation contrastant avec le génial penseur de la jeunesse : le pressentiment d'une mort prochaine aurait donné à ses derniers sermons des accents de simplicité tenant à la conscience de la finitude humaine, qui en appellent moins à l'intelligence de la Loi qu'au Buddha lui-même? On connaît de Dōgen, à la veille de sa disparition, tandis qu'il se rendait à Kyōto sur le conseil de son protecteur, Hatano Yoshishige 波多野義重, le 5 du $8^{\mathrm{e}}$ mois de 1253, un ultime poème à ce dernier invoquant le Tathāgata :

"Au monastère du Eiheiji où je vis depuis dix années,

Me voilà cloué sur mon lit de malade depuis dix mois.

Quittant pour un temps ma montagne afin de m'enquérir d'une médication auprès des hommes,

Le Tathāgata me prend par la main pour m'emmener voir le Roi médecin?"25

Cette hypothèse $^{26}$ rendrait compte de contradictions apparentes dans la pensée de Dōgen. La définition qui y est donnée du cycle de la vie et de la mort comme constitutif de la vie du Buddha ne la rendrait-elle pas plausible?

Ce sermon pose à l'exégète une série de questions. Est-il une profession de foi de son auteur, qui révèlerait une évolution allant d'un "jeune" Dōgen à un "vieux" Dōgen, ou la coexistence de "deux" Dōgen? S'agit-il d'un sermon s'adressant à un adepte laïc? Ou encore a-t-on affaire à un faux? ${ }^{27}$ Dans ce dernier cas, force

25. “Nyorai te wo sazukete iō ni mishimu 如來授手見醫王” : le Kenzeiki 建撕記, biographie de Dōgen composée par Kenzei 建撕 (1415-1474?), dans ses versions de l'ère Tenshō 天正 (1573-1592) et de 1680 (Enpō 延璝, 7), lit ces vers : "te wo sazukete iō ni mamike kitaru ga gotoki" (C'est comme si on me prenait par la main pour m'emmener vers le Roi-médecin). Cette lecture qui semble forcée n'est pas adoptée ici.

${ }^{26}$ Elle est émise par Tamaki Kōshirō 玉城康四郎 (“Dōgen”, Chūōkōron 中央公論, 1983, pp. 90-92; “La lutte à mort du moine Dōgen", in Matsugaoka bunko kenkyū kiyō 松岡文庫研 究紀要, n6, 1992, pp. 141-146). Sans le dater, Kawamura Kōdō 河村孝道 se prononce en faveur de l'authenticité de ce sermon : "Shōbōgenzō seishikan ronkō shingiron o keiki to shite 正法眼蔵生死卷論考一真份論を契機として” [Etude sur le chapitre “Vie et mort” du “Thésaurus de l'œil de la vraie Loi,-A propos de son authenticité-]", in Shūgaku kenkyū 宗 學, ${ }^{\circ}$ 5, avril 1963, pp. 92-99, "Shōbōgenzō kenkyū josetsu - Shōbōgenzō seishi kan no kenkyū (I) 正法眼蔵研究序説・正法眼蔵生死巻の研究” [Introduction à l'étude du “Thésaurus de l'œil de la vraie Loi", recherches sur le chapitre "Vie et mort" du "Thésaurus de l'œil de la vraie Loi"], in Komazawa daigaku bukkyoggakubu kenkyu kiyō 駒沢大學仏教学部研究紀要、 $\mathrm{n}^{\circ} 23$. mars 1965, pp. 108-125; “Shōbōgenzōseiritsushi no kenkyū 正法眼蔵成立史の研究” [Recherches sur l'histoire de la formation du "Thésaurus de l'œil de la vraie Loi], Tōkyō: Shunjūsha 春秋社, 1987, pp. 781-787.

${ }^{27}$ Itō Yüten 伊藤猷典 y a vu un faux (Aichi gakuin daigaku ronsō 愛知学院大学論丵, I et II, 1954, 1955). Nishiari Bokusan 西有穆山 pense que Dōgen aurait rédigé ce sermon pour Shinran (1178-1262), le célèbre amidiste (Shōbōgenzō keiteki 正法眼蔵啓迪 [Guide au Thésaurus de l'œil de la vraie Loi], 1916, Tōkyō: Yoyogi shoin 代々木書院, 1965). Pour Okubo Dōshū, ce texte, sans faire partie intégrante du "Thésaurus", n'en constitue pas moins 
serait d'admettre que le faussaire a réussi un tour de force : sa contrefaçon serait un chef-d'œuvre si parfait, qu'elle aurait réussi à déjouer la tradition presque unanime du Sōtō. Mais cette hypothèse n'est-elle pas à son tour coûteuse à soutenir? Ce faux trahirait dans le Sōtō, à une époque indéterminée, la volonté de capter un auditoire élargi à des adeptes laïcs appartenant aux milieux amidistes ou Zen. Mais un tel mobile est-il compatible avec la tradition éminemment rigoriste du Sōtō? Sans répondre à ces interrogations, je tiens simplement à marquer ici que la place à accorder à ce texte n'est pas indifférente à l'interprétation de la pensée du fondateur du Sōtō.

\section{Traduction $^{28}$ \\ Thésaurus de l'cil de la vraie Loi, version secrète Volume I : "Le cycle de la vie et de la mort 生死."}

Cas du maître de dyäna Damei Fazhang 大梅法常. Une fois que Jiashan 夾山 et Dingshan 定山 pérégrinaient ensemble, Dingshan déclara : "Lorsque dans le cycle de la vie et de la mort il n'est pas de Buddha, le cycle n'est plus." Jiashan rétorqua : "Quand dans le cycle de la vie et de la mort se trouve un Buddha, l'on n'y est plus la proie aux illusions." Nos deux hommes ne cessaient de deviser de la sorte sur la vérité de leurs thèses. Ils escaladèrent alors la montagne dans le but d'aller consulter le maître Damei sur le sujet. Une fois les salutations échangées, Jiashan se risqua à demander: "Des thèses de chacun de nous deux laquelle est la vraie?" Le maître répondit : “Qui colle une fois à la vérité s'en éloigne unc autre." Jiashan s'enquit à nouveau : "Mais laquelle donc de nos deux

\footnotetext{
un sermon indépendant dont la paternité à Dōgen ne peut être mise en doute, pour des raison de contenu. Les variantes des deux versions dont on dispose-celle du Eiheiji et la version de la tradition de Menzan qui ajoute le dialogue en chinois-, tiendraient au fait qu'on en aurait révisé ou non le texte primitif. Son intégration dans le "Thésaurus secret" daterait de l'époque Ashikaga (Dógen zenjiden no kenkyū 道元禪師傳の研究 [Recherches sur la biographie du maître de dhyāna Dōgen], Tōkyō: Iwanami shoten 岩波書店, 1953, pp. 357358). Les partisans de son authenticité suivent en général l'interprétation de Honkō (1770) : "Les versions du "Thésaurus" en 75 fascicules ou en 80 fascicules sont sans ordre établi et nombreux en sont les chapitres [?] douteux. Toutefois, comme on a tiré ce volume après coup du grenier du Lihheiji comme de derrrière les fagots, sa date n'a pas été notée : mais comment pourrait-on entretenir des doutes concernant le texte?" "Quand on regarde ce volume du début à la fin, on peut se demander s'il n'a pas été montré à quelque fonctionnaire. Dans la superficialité de son texte, il est très proche de la vérité profane, et quant au fond même. un authentique dragon n'arriverait pas aisément à rivaliser avec lui. A combien plus forte raison une personne qui aimerait en vain sculpter le dragon [travailler le style]." (Shōhōgenzō chükai zensho 正法眼蔵註解全書, IX, pp. 456 et 458).

${ }^{2 \times}$ Je suis l'édition établie par Etō Sokuō 衛藤即雇, Iwanami bunko 岩波文庫, volume III, 1943. pp. 239-241; v. Ōkubo Dōshū, 1989, pp. 779-780.
} 
thèses colle à la vérité?" Le maître jeta en guise de réponse : "Vas-t-en, et reviens me voir demain." Jiashan revint le lendemain et posa à nouveau sa question. Le maître répliqua : "Qui colle à la vérité ne pose pas de question, et qui en pose en est déjà bien loin." Jiashan resta interdit et déclara par la suite : "A demeurer maintenant chez le maître Damei, j’ai perdu mon œil unique!",29

Lorsque dans le cycle de la vie et de la mort il se trouve ${ }^{30}$ un Buddha, le cycle n'est plus. On déclare de même : quand dans le cycle de la vic ct de la mort il n'est plus de Buddha, on n'erre plus dans ce cycle.

Ces thèses ${ }^{31}$ ont été émises par Jiashan ${ }^{32}$ et Dingshan ${ }^{33}$; ces paroles reviennent donc à ces deux maîtres de dhyāna. Venant de personnes accomplies dans la voie, elle ne peuvent à n'en point douter avoir été proférées en vain. ${ }^{34}$

\footnotetext{
${ }^{29}$ Cette anecdote est rajoutée en prologue dans la version du "Thésaurus" dite de Naippō, de Fuyō Rōran, en date de 1791, dans la tradition de Menzan (1683-1769). On la trouve presque terme à terme dans le "Recueil de la transmission de la lampe 景德傳燈錄” ( $T$. LI, $n^{\circ}$ 2076, pp. 254c-255a) ainsi que dans les "Dicta de Fazhang" copiés au XIII ${ }^{c}$ siècle ("Matériaux conservés à la Bibliothèque de Kanazawa 金沢文庫資料全書, Ouvrages bouddhiques 伀典, I, Textes Zen 禅籍篇”, 1974, p. 14ab). V. Shōbōgenzō chükai shinshū 正法 眼蔵䚾解新集 [Nouveau recueil de commentaires au Thésaurus de l'œil de vraie Loi] de Nagahisa Gakusui 永久岳水, Tōkyō: Yoyogi shoin , 1931, pp. 23-24.

Sur l'œil unique, v. "Nature de Buddha" de 1241 : quand on voit la nature de Buddha dont on est porteur, on ouvre l'œil unique [de la sapience], mais quand c'est l'esprit humain ayant déjà épanoui la nature de Buddha, qui regarde à nouveau la nature de Buddha, il perd cet ail unique. Il perd alors l'unicitité et la simplicité de son regard en le dédoublant inutilement et en hypostasiant l'objet de sa quête : "Quand on voit sa nature de Buddha on ouvre son œil unique, mais quand c'est la nature de Buddha [qui se voit elle-même], on perd son œil unique".

"Naippō porte respectivement : "il ne se trouve pas de Buddha", et "il se trouve un Buddha". Elle est philologiquement correcte si l'on se reporte au texte chinois cité en prologue dans Naippō. La version "secrète" du Eiheiji s'en est écartée. Nous laissons telle quelle la leçon du Eiheiji qui est la plus populaire.

${ }^{31}$ Kokoro : le sens de cette assertion. Naippō : kore, cela

${ }^{32}$ Damei Fazhang (752-839) est un disciple de Mazu 馬祖 (709-788) qui a séjourné au Mont Damei, situé dans la préfecture de Ningbo 寧波 (actuel Zhejiang) en 796, afin d'y faire retraite pour une quarantaine d'années. On a de lui un recueil de ses propos en un volume, les "Dicta du maître de dhyāna Fazhang du Mont Damei", qui a été composé en 1004 au plus tard.

${ }^{33}$ Shanhui 善會 du Jiashan 夾山 (805-881) se mit à l'école de Zhuanzi Dezheng, alors capitaine de vaisseau au Zhezhong 浙中 (actuel Jiangsu), sur les conseils de Yuanzhi de Daowu 道吾円智 (769-835). En 870, il s'installe au Jiashan (actuel Hebei) et s'y adonne au chan de l'école de Yueshan 樂山. Shenying 神英 du Dingshan 定山 (?-?) est un moine de l'époque des Tang. Disciple de Weishan Lingyou 偽山籃祐 (771-853), il a résidé au Mont Dingshan dans le Tuzhou 余州 (actuel Anhui).

${ }^{34}$ Maukeji. Naippō et Kōzen 晃全 (1729): mahukeji.
} 
Qui veut se détacher du cycle de la vie et de la mort a, en vérité, à tirer au clair ces thèses. Que d'aucuns ${ }^{35}$ aillent chercher le Buddha en dehors du cycle transmigratoire, c'est à peu près comme s'ils mettaient le timon au septentrion afin de se diriger vers le pays de Yue [situé au sud], ${ }^{36}$ ou comme s'ils faisaient face au méridien pour voir l'étoile polaire. Ils ne feraient guère qu'accumuler des causes les plongeant dans la transmigration et perdre de vue plus encore la voie de la délivrance. Mais s'ils s'avisent que le cycle de la vie et de la mort n'est, tel quel, autre que le nirvāna, ils n'auront rien à haïr en tant que cycle de la vie et de la mort ni rien à souhaiter en tant que nirvāna $a^{37}:$ dans cet état, ils seront enfin détachés en regard de la vie et de la mort.

C'est une erreur de croire qu'on passe de la naissance à la mort. La naissance est un état momentané qui a d'emblée ${ }^{38}$ un avant et un après. On soutient pour ce motif dans l'enseignement bouddhique que la naissance ${ }^{39}$ n'en est pas une. L'extinction de même est un état instantané qui lui aussi ${ }^{40}$ a un avant et un après. Partant, on professe que l'extinction n'en est pas une. Au moment où l'on parle de naissance, ${ }^{41}$ il n'est rien qui soit en dehors d'elle, et à l'instant où il est question d'extinction, ${ }^{42}$ il n'est rien qui se trouve en dehors d'elle non plus. ${ }^{43}$ Voilà bien pourquoi, pour autant que la naissance survienne, il n'y a qu'elle, et dès que la mort approche, il ne reste plus qu'à s'employer en vue d'elle. ${ }^{44}$ Il n'y a donc rien à haïr ni rien à souhaiter. ${ }^{45}$

3is Hito; Naippō : manque.

3t V. "Discours sur l'application à la voie" (traduction B. Faure, p. 89) : réciter des formules en espérant atteindre un éveil [extrinsèque à la nature humaine] c'est mettre le timon au septentrion pour se diriger vers le pays de Yue ou tenter la quadrature du cercle.

${ }^{37}$ C'est une reprise mot pour mot d'une phrase de Saichō (766-822) sur l'identité du nirvāna et du samsāra ("Recueil essentiel sur la doctrine de la Tête de bœuf de l'Ecole du Sūtra du Lotus de la Loi sublime du Tendai 天台法華宗牛頭法門要策”; CEuvres complètes, V, p. 61). V. Tamura Yoshirō 田村芳郎 (“Etudes sur le nouveau bouddhisme de l'époque de Kamakura 鎌倉新仏教の研究”, Kyōto, 1965, p. 549), pour qui le sermon de Dōgen exprime ici l'idée propre à la doctrine de l'éveil foncier qui avait cours au Hieizan, selon laquelle le nirvāna n'est pas extérieur au samsāra et la réalisation en dehors de la pratique. L'auteur ne met aucunement en cause l'authenticité du présent sermon. On pourrait songer à un souvenir de lecture du jeune Dōgen ou à l'intervention d'un moine du Tendai.

${ }^{38}$ Sude $n i$ : absent dans une version.

${ }^{34}$ Shō 生; version secrète : shōshō : les naissances.

4" Mata : aussi; absent dans une autre version.

"Membre de phrase manquant dans une autre version. Naippō lit toki ni au lieu de toki niwa.

${ }^{42}$ Tokiwa. Naippō lit toki ni.

${ }^{43}$ Metsu no hoka ni mono nashi. Naippō lit metsu yori hoka ni mono nashi.

${ }^{44}$ Tsukau : s'employer à; ou tsugau : assurer un lien avec. Honkō (1770) propose la lecture tsutohu, s'assembler, qui semble moins satisfaisante. Naippō lit la fin de la phrase naku au lieu de nakare, ce qui ne change guère le sens de la phrase.

${ }^{45}$ Nous suivons la leçon du Eiheiji, itohu koto nakare : ne haïssez point, et non Naippō : ihu koto nakare: ne dites point que, il n'y a pas à s'employer en vue de. 
Le cycle de la vie et de la mort constitue la vie du Buddha. Si, prenant en aversion ce cycle on cherche à le rejeter, on ne fera que perdre la vie du Buddha. Lorsqu'on s'attache à ce cycle en y stationnant, on passe à nouveau à côté de la vie du Buddha ${ }^{46}$ : on donnera un coup d'arrêt à la manière d'être du Buddha. C'est au moment où l'on est dénué d'aversion et de désir qu'on se tient ${ }^{47}$ pour la première fois dans la pensée du Buddha. Prenons cependant garde à ne rien supputer avec la pensée ni à ne rien exprimer au moyen du langage. La sculc chose qui compte est d'oublier son corps et sa pensée en s'en détachant, afin d'aller se précipiter dans la famille du Buddha, se laisser œuvrer par lui et le suivre. A ce moment-là, on se détachera du cycle de la vie et de la mort et deviendra Buddha, sans appliquer de force $\mathrm{ii}^{48}$ employer sa pensée. Qui donc à ce compte-là éprouverait de l'ennui dans sa pensée ? $^{49}$

Pour qui veut devenir Buddha, il est une voie extrêment aisée. Ne faire aucun mal, ne pas s'attacher au cycle de la vie et de la mort, éprouver une profonde compassion à l'endroit de tous les êtres, révérer ceux qui sont audessus de nous et prendre en compassion ceux qui sont en-dessous, ne rien haïr ni désirer en sa pensée, ${ }^{50}$ n'avoir aucun objet d'attachement ni sujet d'affliction en sa pensée. Voilà ce qu'on appelle l'état de Buddha. Il n'est rien dont il y ait à s'enquérir de plus.

[Colophon :] ${ }^{51}$ On ne voit pas que ce rouleau ait été diffusé d'ordinaire de par le monde. Dans la bibliothèque du Eiheiji, il est depuis jadis trois versions du "Thésaurus de l'œil de la vraie Loi". Parmi elles, dans une version réunie en trois fascicules, se trouve ce chapitre sur le Cycle de la vie et de la mort qui est le deuxième du premier fascicule. C'est lui que je viens de recopier.

\footnotetext{
4h On inochi : l'auguste vie; Honkō omet l'honorifique on-.

${ }^{47}$ Honkō : itaru, on atteindra; Naippō : iru nari : on sera, se tiendra.

${ }^{48}$ Naippō et Honkō lisent mo : même, aussi, inutile ici.

${ }^{4 y}$ Cette phrase manque dans Naippō et Honkō.

"Naippō lit fautivement ari à la place de nakute.

s! Version de Kōzen conservée au Eiheiji, copiée par Taikyō 台橋 en 1729 (Kyōho 享保, 14).
} 